\title{
AVALIAÇÃO DA AUTO-ESTIMA EM JOVENS TRANSMONTANOS
}

\author{
SELF-STEEM AMONG THE YOUTH OF TRÁS-OS-MONTES
}

\author{
José Vasconcelos RAPOSO* \\ Carlos Avelens FREITAS
}

\begin{abstract}
RESUMO
Em Trás-os-Montes e Alto Douro as cidades são, essencialmente, pequenas com uma população estudantil proveniente do meio rural e da urbe. Devido a estas características, considerámos importante levar a cabo um estudo que nos desse uma idéia clara sobre a auto-estima desses jovens, principalmente os adolescentes. Este trabalho é um estudo exploratório e descritivo. Pretendemos saber qual é o nível de auto-estima de jovens escolarizados, com idades compreendidas entre os 14 e os 16 anos de idade de ambos os sexos e se existe alguma relação entre esses níveis e: local de residência habitual (aldeia versus cidade); sexo e as idades.

A amostra foi constituída por 1033 jovens de ambos os sexos (547 raparigas 444 rapazes). Estes foram, por sua vez, divididos em grupos consoante o seu local de residência: Aldeia 460 (241 femininos e 219 masculinos) e Cidade 573 (306 femininos e 267 masculinos). Para calcular a auto-estima recorremos à escala de Rosenberg, respeitando a sua escala inicial - Gutman. Os testes estatísticos utilizados foram a correlação de Pearson incluindo o Chi-quadrado.

Os resultados demonstram existir diferenças por sexo e grupo etário relativamente ao local de residência. Os moradores nos centros urbanos apresentaram maiores índices de auto-estima comparativamente aos residentes em zonas rurais. No entanto, os últimos parecem apresentar níveis mais uniformes de auto-estima do que as raparigas, sendo esta diferença mais acentuada nos meios rurais.
\end{abstract}

Palavra Chave: auto-estima, urbano e rural

\section{ABSTRACT}

The present study investigated whether area of residence, age group and sex explain difterences in the self-esteem of boys and girls.

(*) Pró-Reitor da Universidade de Trás-os-Montes e Alto Douro.

Endereço para correspondência: UTDA - Mirada do Douro - R. D. Diniz 5210 - Mirada do Douro - Portugal - E-mail: jraposo@utad.pt 
The sample consisted of 1033 students of both sexes (444 boys 547 girls) were grouped into two residential area groups village $(N=460)$ with 241 girls and 219 boys and city ( $N=573)$ with 306 girls and 267 boys. The Rosenberg self-esteem scale was used following the author's initial recommendation to treat it as a Guttman scale

The Chi-square demonstrated that there was significant differences between the ages and sex. Residents in the urban areas presented higher levels of self-esteem than rural residents Boys seem to present more uniform levels of self-esteem than girls among whom the greater discrepancies were found in the group living in the rural areas.

Key words: Self-esteem, rural, urban

\section{INTRODUÇÃO}

O Presente trabalho insere-se num conjunto de outros que têm vindo a ser desenvolvidos sob orientação de Raposo, com o intuito de avaliar os níveis de auto-estima entre as populações portuguesas. Vasconcellos (1998) recorda que este é um dos conceitos mais utilizados em vários domínios da vida das pessoas, mas na realidade ainda não temos uma boa compreensão sobre a sua complexidade. Na literatura da especialidade há alguma consistência na demonstração que a auto-estima tende a estar correlacionada positivamente com a actividade física (Kincey et al., 1993), local de residência (Daly, Kreiser, \& Roghaar, 1994), sexo, saúde (Sullivan, Kessler, \& Kendler, 1998; Baldwin, Courneya, 1997), sucesso escolar (KeltikangasJarvinen, 1992; Prawat, Grissom e Parish, 1979), entre outros. O estudo que levamos a cabo em três cidades de Trás-os-Montes (Chaves, Mirandela e Vila Real) procura saber se a área residencial, o sexo e a idade são diferenciadores de níveis de auto-estima em jovens com idades compreendidas entre os 14 e os 16 anos de idade.

\section{Delimitação Conceptual}

A auto-estima (AE) tem ocupado uma posição central na explicação do comportamento humano (Fox e Corbin, 1989). É aceite que esta é um indicador do bem estar mental (ajuste emocional e saúde mental) e um mediador do comportamento humano (Fox, 1988). Hines e Groves (1989) afirmam que vários investigadores são de opinião que um auto-conceito (AC) positivo é fundamental para o desenvolvimento e ajustamento dos jovens aos dias de hoje. Estes mesmos autores referem que para Purkei et al. (1973) uma AE positiva é reflectida num crescimento e desenvolvimento também positivo. Batista (1995) é de opiniãoque uma das razões que justifica o extraordinário interesse na AE global é que esta assume uma importância relevante no comportamento psicológico do indivíduo. Esta autora reforça a sua opinião referindo que uma baixa AE parece estar associada com a depressão, ansiedade e baixa satisfação com a vida. Também Burns (1979) havia chegado à mesma conclusão. Outros autores (i.e. Daly, Kreiser, \& Roghaar, 1994) argumentam que a auto-estima está relacionada com a área residencial, classe social (Sullivan, Kessler, \& Kendler, 1998; Simon, 1972; Smith et al., 1973), etc.

A auto-estima (AE) e o auto-conceito (AC) são vistos como predictores do comportamento humano tanto no domínio académico como em actividades anti-sociais (Calfas e Taylor, 1994), podendo concluir-se que uma baixa $A E$ terá repercussões negativas no dia-a-dia do indivíduo, nos vários domínios da sua realização.

Smelser (1989) sugere que a maior parte dos problemas actuais da sociedade têm a sua origem na baixa $A E$ dos cidadãos. Os indivíduos que acreditam que não são válidos para a so- 
ciedade tendem a refugiar-se em comportamentos improdutivos, dispendiosos, desviantes e perigosos para esta. Segundo este autor, tornase essencial para os líderes da sociedade, estabelecerem condições sociais para maximizar o desenvolvimento da AE da população estabelecendo programas que ajudem a reabilitar os cidadãos provenientes de famílias e comunidades que se considerem de baixo valor. Bhatti et al. (1989) após reverem a literatura que aborda a relação entre a $A E$ e os maus tratos infantis, verificaram que são necessários mais dados para interpretar a natureza desta possível relação. No entanto, a AE parece estar relacionada com os maus tratos infantis, pois quer as crianças que os sofrem, quer os pais que os infligem, têm a tendência a apresentar baixos níveis de $\mathrm{AE}$.

Em relação à gravidez na adolescência, uma $A E$ elevada pode levar os jovens a iniciaremse mais tarde nas relações sexuais (Crockenberg e Soby, 1989). Paralelamente, os jovens com insucesso escolar parece envolverem-se em relações sexuais mais cedo. No entanto, e segundo os mesmos autores, as razões que levam os jovens com baixa e com alta $A E$ a iniciarem-se nas relações sexuais são diferentes.

Sendo o desemprego um dos principais problemas das sociedades de hoje, existem poucas indicações para que uma baixa $\mathrm{AE}$ seja um obstáculo para a aderência ao emprego (Schneiderman et al. 1989). No caso dos dependentes de álcool e drogas, parece ser preferível desenvolver estruturas da personalidade que contribuam para o aumento da AE do que procurar influenciar directamente esta; ou seja, é preferível desenvolver nos indivíduos a capacidade para auto-melhorarem a sua AE (Skager e Kerst, 1989).

Além da AE existem outros conceitos e formas de abordagem do "eu": auto-aceitação, autoconfiança, auto-imagem, autovalor, auto-respeito, auto-avaliação, auto-satisfação, etc. Semelhanças entre alguns destes conceitos têm sugerido aos autores usá-los indiscriminadamente, o que conduz a uma confusão conceptual e metodológica, surgindo, conseqüentemente, a necessidade de os diferenciar entre si. De todos os citados, os que com mais facilidade se confundem, até porque há autores (Shavelson et al. 1976) que os utilizam de forma indiscriminada, são a $A E$ e o auto-conceito (AC). Nos últimos anos, a distinção entre estes dois conceitos tem sido geradora de controversa e polémica académica (Wylie, 1979).

Fox (1988) define o AC como uma capacidade descritiva em que afirmações como "eu sou um atleta", "eu sou um estudante" são aglomeradas, produzindo um conhecimento multifacetado do próprio "eu". Por sua vez, a AE tem uma com ponente avaliativa e pode ter por base várias avaliações específicas de uma situação, tal como, por exemplo, "eu sou o melhor corredor da minha turma".

Harter (1983) é de opinião que os aspectos descritivos e avaliativos do AC distinguem-se claramente no quadro conceptual e empírico, e que ambos se complementam, pois para avaliar é necessário conhecer primeiro a percepção que o indivíduo tem no domínio em que faz essa avaliação. Segundo este autor, tanto o AC como a AE são com ponentes da auto-percepção, referindo-se $o \mathrm{AC}$ aos aspectos conotativos e a $\mathrm{AE}$ aos aspectos avaliativos.

Segundo Weiss (1987), o $A C$ refere-se às descrições ou rótulos que o indivíduo traz consigo, tais como atributos físicos. características comportamentais ou qualidades emocionais e a $A E$ refere-se à componente avaliativa e afectiva do AC, ou seja, reflecte os julgamentos qualitativos e sentimentos que cada um tem face à descrição que faz de si. Para Weiss (1993), esta definição sugere que o nível de AE da criança pode ser identificado pela sua comunicação verbal, tal como atribuições para o sucesso ou fracasso, ou através de comportamentos não verbais, tais como participação ou rejeição da actividade física, esforço ou preguiça, persistência ou desistência na aprendizagem de novas habilidades. Watkins e Dhawan (1989, citados por Greene e Reed, 1992) partilham da mesma opinião. Estes autores consideram que os aspectos avaliativos e descritivos do "eu" estão relacionados, não podendo ser considerados sinónimos. 
Num estudo de Hines e Groves (1989) a AE é considerada uma com ponente do $\mathrm{AC}$ baseado no desempenho. É uma atitude de aprovação ou desaprovação consigo próprio e indica o grau em que cada um acredita ser capaz, importante, bem sucedido e digno. Ponte et al. (1991) definem o AC como a "percepção que o indivíduo tem de si próprio (como ser físico, social ou moral), o juízo que devido a isso, forma de si" (p.207). Por sua vez, Whitehead (1993) e Serra (1986) também distinguiram o AC da AE, considerando o primeiro como sendo "as auto descrições que cada um faz acerca daquilo que é", e o segundo como a "avaliação que cada pessoa faz de bom ou de válido acerca dessas percepções" (p. 132). Noutros casos, estes termos são usados indiferentemente (Shavelson et al. 1976) porque a avaliação e o afecto parecem ser uma conseqüência natural da auto-descrição.

Resumindo as idéias anteriores, Schilling (1986, citado por Lopes 1996) destingue clara e concretamente os dois conceitos da seguinte forma: "o nosso AC são as percepções que temos de nós próprios em várias áreas - como nos percebemos física, intelectual e socialmente. A nossa AE é determinada pelas avaliações que pomos nessas percepções" (p. 301).

Haywood (1986), define a AE como um julgamento pessoal das suas próprias capacidades, importância, sucesso e mérito e exprime-se aos outros em palavras e acções. Weiss (1987) cita a definição de AE apresentada por Coopersmith (1959) considerando-a a mais relevante para a actividade física e desporto:

" $A$ avaliação que um indivíduo faz e que habitualmente mantém quando se observa a si próprio: expressa uma atitude de aprovação ou desaprovação e indica a dimensão da crença que o indivíduo tem em ser capaz, significativo, bem sucedido e com valor. Em resumo a $A E$ é um julgamento pessoal de valor que é expresso em atitudes que o indivíduo comunica aos outros através de informação verbal e outros comportamentos expressivos".

Mais recentemente, Godin e Leval (1993) definem a $A E$ como a medida de aprovação ou desaprovação sentida pela própria pessoa; é a crença na sua habilidade para o sucesso e no seu valor social e pessoal que se traduz na forma como o indivíduo fala e age no seu dia-a-dia.

De uma forma geral, os autores consideram a $\mathrm{AE}$ e o $\mathrm{AC}$ como componentes da autopercepção, sendo o primeiro a avaliação da informação contida no segundo. Parece ser também evidente que quando se fala subentende-se a existência de uma avaliação pessoal das suas próprias capacidades que é expressa aos outros em palavras e atitudes.

A auto-estima pode definir-se, segundo Jaquish e Savin-Williams (1981), como a imagem global, positiva ou negativa, que um indivíduo faz de si próprio, numa determinada situação. Ainda segundo Rosenberg (1989), a autoestima é a atitude negativa ou positiva em relação ao "self'. A importância do estudo dos fenómenos da auto-estima nos jovens reside principalmente no facto de valores baixos de auto-estima estarem relacionados, segundo Rosenberg (1989), com sentimentos de: depressão, infelicidade, tristeza e inferioridade. É, assim, necessário conhecer bem essas relações de forma a possuirmos uma idéia clara desses problemas entre os jovens.

Segundo Schilling (1986), níveis altos de auto-estima estão relacionados, em primeiro lugar, com a forma como nos sentimos "capazes", isto é, com a consciência de que possuímos capacidades que concorrem para os nossos objectivos. Em segundo lugar, com a necessidade de nos sentirmos significantes, portanto que aquilo que sentimos, dizemos ou fazemos influencia as pessoas que nos rodeiam. Em terceiro lugar, precisamos de nos ver a nós próprios como potencialmente poderosos, na medida em que temos algum controle sobre o que nos acontece e somos, portanto, capazes de tomar decisões e fazer opções. Para Rosenberg (1989), níveis altos de auto-estima estão associados a um indivíduo que tem respeito próprio, se considera útil, não necessariamente superior aos outros, mas também não inferior, o que reconhece as suas limitações e anseia crescer e melhorar-se como pessoa, assim como as suas condições de vida. Por outro lado, 
Rosenberg (ibid) considera que níveis baixos de auto-estima estão relacionados com a insatisfação pessoal, sentimentos de rejeição e repúdio pessoal, em que o indivíduo considera o seu 'self' desagradável e gostaria que ele (o "self') fosse diferente.

Para além destes aspectos há, ainda, evidência empírica que os indivíduos com níveis baixos de auto-estima tendem a ter piores índices de saúde do que os outros (Beer, 1987). Ainda Relacionado ao componente saúde temos a actividade física. A relação entre a actividade física e a auto-estima é talvez a correlação mais estável que se tem encontrado nos estudos realizados nesta área do saber (ver Coelho e Raposo, 1998).

Com o presente estudo pretendemos avaliar os níveis de auto-estima dos jovens de três cidades de uma das regiões, que ao longo da sua história, mais tem sido discriminada pelos governos que se têm sucedido. A região de Trás-osMontes é uma das que mais sofreu o impacto da emigração e onde presentemente mais se tem feito sentir a desertificação do interior do país.

Em Trás-as-Montes e Alto Douro as escolas têm uma particularidade interessante. Como as cidades que existem são, essencialmente, pequenas e rurais, as nossas escolas são uma amálgama de jovens provenientes quer da cidade quer da aldeia. Devido à essas característi- cas, considerámos importante iniciar um trabaIho que nos desse uma idéia mais clara sobre auto-estima desses jovens, principalmente os adolescentes.

Com este trabalho realizámos um estudo exploratório e descritivo, na área da auto-estima, em três escolas. Procurámos saber qual o nível de auto-estima de jovens escolarizados, com idades compreendidas entre os 14 e 16 anos (inclusive) de ambos os sexos e se existe alguma relação entre esses níveis e: asua residência habitual (aldeia versus cidade), assim como entre o sexo e as idades.

\section{Material e Métodos}

Neste estudo recorremos ao paradigma das correlações.

Para o propósito do presente estudo utilizámos uma amostra de conveniência, proveniente de cinco escolas, uma de Mirandela, duas de Chaves e duas de Vila Real. O número de alunos das diferentes escolas das cidades de Vila Real e Chaves contribuíram com percentagens aproximadas de $50 \%$ para a amostra final dessas cidades. Pretendíamos, assim, reduzir de alguma forma as diferenças de carácter sociodemográfico que poderiam ocorrer pelo facto das amostras pertencerem a uma só escola da cidade.

\begin{tabular}{|c|c|c|c|c|c|c|c|c|c|c|c|c|c|c|c|c|}
\hline \multirow[b]{3}{*}{ IDADES } & \multicolumn{7}{|c|}{ ALDEIA } & \multicolumn{8}{|c|}{ CIDADE } & \\
\hline & \multicolumn{3}{|c|}{ Feminino } & \multicolumn{4}{|c|}{ MASCULINO } & \multicolumn{4}{|c|}{ Feminino } & \multicolumn{4}{|c|}{ MASCULINO } & \multirow{3}{*}{ TOTAI } \\
\hline & 14 & 1516 & TOTAL & 14 & 15 & 16 & TOTAL & 14 & 15 & 16 & TOTAL & 14 & 15 & 16 & TOTAL & \\
\hline \multirow[t]{2}{*}{ CHAVES } & 14 & \begin{tabular}{|l|l|}
23 & 13 \\
\end{tabular} & 50 & 13 & 19 & 21 & 53 & 37 & 24 & 9 & 70 & 28 & 21 & 23 & 72 & \\
\hline & \multicolumn{3}{|c|}{ ALDEIA } & \multicolumn{4}{|c|}{103} & \multicolumn{4}{|c|}{ CIDADE } & \multicolumn{4}{|c|}{142} & 245 \\
\hline \multirow[t]{2}{*}{ MIRANDELA } & 19 & 2730 & 76 & 11 & 33 & 23 & 67 & 63 & 54 & 58 & 175 & 47 & 49 & 34 & 130 & \\
\hline & \multicolumn{3}{|c|}{ ALDEIA } & \multicolumn{4}{|c|}{143} & \multicolumn{4}{|c|}{ CIDADE } & \multicolumn{4}{|c|}{305} & 448 \\
\hline \multirow[t]{2}{*}{ VILA REAL } & 38 & 4532 & 115 & 36 & 40 & 23 & 99 & 26 & 21 & 14 & 61 & 34 & 20 & 11 & 65 & \\
\hline & \multicolumn{3}{|c|}{ ALDEIA } & \multicolumn{4}{|c|}{214} & \multicolumn{4}{|c|}{ CIDADE } & \multicolumn{4}{|c|}{126} & 340 \\
\hline TOTAL & 71 & \begin{tabular}{|l|l|}
95 & 75 \\
\end{tabular} & 241 & 60 & 92 & 67 & 219 & 126 & 99 & 81 & 306 & 109 & 90 & 68 & 267 & \\
\hline & & & OTAL A & LDEI & IA & 460 & & & & $\mathrm{TO}$ & TAL CIL & DADE & 573 & & & 1033 \\
\hline
\end{tabular}

Quadro 1 - Caracterização da amostra 
Para a recolha de dados foi utilizado um inquérito adaptado de Rosenberg (1989). Este inquérito, de resposta de cruz, era constituído por nove afirmações (itens), a que o inquirido deveria responder se: concordava, concordava plenamente, discordava ou discordava plenamente. No cabeçalho teria, ainda, de responder (de cruz) a três variáveis: idade (14,15e 16anos); sexo (feminino/masculino) e residência (aldeia! cidade).

Segundo Rosenberg (1989), este inquérito tem quatro vantagens:

- é de fácil administração, de forma que se podem utilizar amostras suficientes para análises de multivariância;

- efectua-se rapidamente, o que é uma vantagem quando se pretende a cooperação das autoridades escolares;

- permite classificar numa escala linear desde os que revelam níveis muito baixos de auto-estima aos que revelam níveis muito altos de auto-estima;

- todos os itens estão directamente relacionados com a dimensão do fenómeno que se pretende estudar.

No inquérito os itens "positivos" estão misturados com os itens "negativos" de forma a reduzir a probabilidade do inquérito ser preenchido de forma padronizada, tornando-se assim mais fácil para quem faz o tratamento dos dados detectar e eliminar os inquéritos que não foram preenchidos com seriedade.

Os dados foram informatizados através de um formulário, construído por nós para o efeito, num programa de bases de dados (Microsoft Access 95). Esses dados foram posteriormente exportados para uma folha de cálculo (Microsoft Excel 95) de forma a serem analisados estatisticamente. Para a análise estatística foi utilizado também o programa - Systat 5.03 for Windows.

Os dados depois de informatizados foram traduzidos para uma escala linear de zero a cinco (0-5) adaptada de Rosenberg (1989), em que a zero correspondem os valores mais altos de auto-estima e que vão diminuindo progressivamente consoante os valores se aproximam de cinco (valor mais baixo de auto-estima). A escala original é uma escala de zero a seis (0-6) que possui dez itens. Como o décimo item não possui uma tradução coerente e objectiva para português, depois de várias tentativas de coerência de tradução, decidimos não incluir no questionário a aplicar. Felizmente o item em causa concorre isolado para obtenção da escala, obtendo-se uma escala mais reduzida mas com aproximadamente a mesma precisão. Os itens não concorrem todos da mesma forma para a obtenção dos valores 1 que constituem a escala, por outras palavras não são todos valorizados da mesma forma.

Alguns contribuem de forma directa para a escala e outros só são considerados através de combinações com outros itens. E respeitando a escala de Gutman apresentada por Rosenberg (1989) concorrem de forma directa para a escala os seguintes itens:

1- No geral estou satisfeito/a comigo mesmo/a-em que se o inquirido responder que concordo plenamente ou concordo, é atribuído o valor 0 e se responder que discordo ou discordo plenamenteé atribuído o valor 1.

8- Eu gostava de termais respeito por mim mesmo/a - em que se o inquirido responder que concordo plenamente ou concordo, é atribuído o valor 1 , e se responder que discordo ou discordo plenamente é atribuído o valor 0 .

Combinam-se os itens:

2- Por vezes penso que não sou nada bom/boa e 6- Eu sinto-me por vezes inútil, em que se o inquirido responder a qualquer uma delas concordo plenamente ou concordo é atribuído o valor 1 e se responder às duas simultaneamente que discordo ou discordo plenamente é atribuído o valor 0 ; 
3 - estou apto/a para fazer coisas tão bem como a maioria das pessoas e 5- Sinto que não tenho muito de que me orgulhar, em que é atribuído o valor 0 caso o inquirido responda simultaneamente discordo ou discordo plenamente ao item 5 e concordo plenamente ou concordo ao item 4, caso essa condição não se verifique, é atribuído o valor 1 .

4 - Sinto que tenho um bom número de qualidades, 7 - Eu sinto que sou uma pessoa com valor, pelo menos num plano de igualdade com os outrose 9em termos gerais estou inclinado/a a sentir que sou um/a falhado/a, em que é atribuído o valor 1 caso se verifiquem todas as seguintes condições ou pelo menos duas delas): o inquirido respon- da que discordo ou discordo plenamente aos itens 3 e 7 e responda que concordo plenamente ou concordo ao item 9. Caso estas condições não se verifiquem é atribuído o valor 0 .

Os valores atribuídos são somados de forma a obter-se um valor total para cada inquérito $($ total $=$ Valor item $1+$ Valor $($ item2;6) + Valor (item 4;5) + Valor (item 3;7;9) + Valor item 8).

A escala de zero a cinco (0-5) foi, por sua vez, transformada numa outra, em que somando os valores 0 e 1 da anterior se obtinha - Alta (auto-estima); somando 2 e 3 se obtinha - Média (auto-estima) e somando 4 e 5 se obtinha-Baixa(auto-estima) (Rosenberg, 1989) (fig. 1). Os dados foram analisados recorrendo quer a uma quer a outra escala, consoante as necessidades da análise.

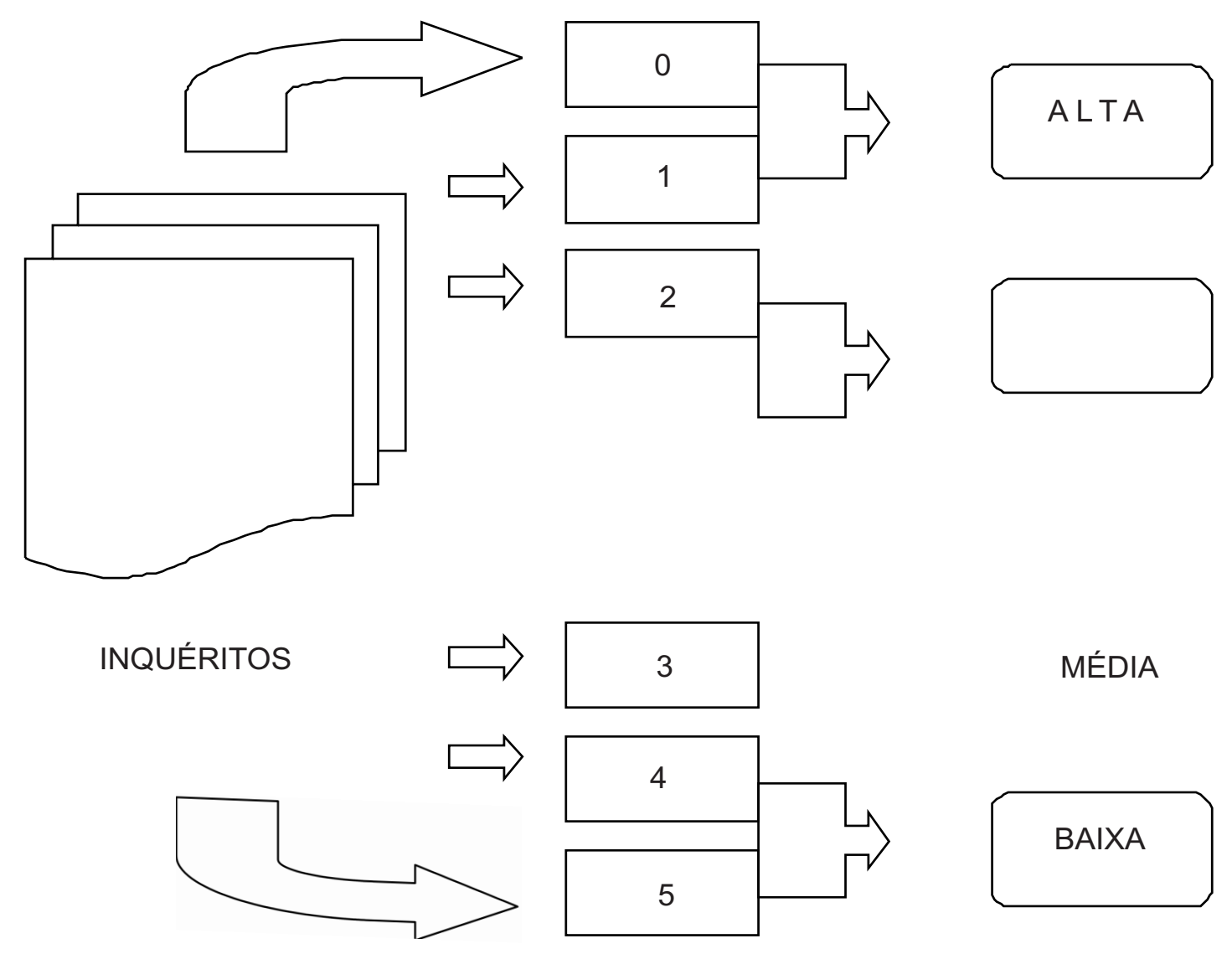

Figura 1 - Escalas utilizadas 
As escalas apresentam valores em termos de percentagens que foram calculados em termos de frequência.

Os inquéritos foram submetidos aos estudantes para preenchimento por sete professores de Educação Física (dois em Chaves; dois em Vila Real e três em Mirandela), a quem explicámos, pessoalmente, a dinâmica dos mesmos e a forma de os apresentar, assim como os treinamos na técnica de "probing", de modo a poderem explicar aos alunos como os deveriam preencher sem Ihes avançarem qualquer indicação sobre a escolha da resposta. Os inquéritos foram preenchidos e recolhidos nos cinco minutos iniciais das aulas de Educação Física. Foi explicado aos alunos que o inquérito era anónimo e que no quadro das idades (14, 15 e 16 anos) deveriam preenchê-lo de acordo com a idade actual (nesse dia), no quadro da morada (aldeia e cidade) deveriam colocar a residência habitual mesmo que esse ano tivessem mudado de residência e que caso morassem numa vila deveriam incluirse nos que moram na cidade. Não estabelecemos nenhum tempo limite de residência obrigatória para esta ser considerada como residência habitual, conscientes de que isso iria introduzir um factor de erro no nosso estudo, mas assumindo esse erro como resultante da necessidade de possuir um inquérito de fácil explicação e rápido preenchimento.

A análise dos resultados seguiu os passos utilizados e recomendados por Rosenberg (1989) em termos de processamento estatístico. Assim utilizámos uma análise de frequência e $X^{2}$, em que analisámos a amostra total, a amostra só dos alunos que moravam na cidade e a dos alunos que moravam só na aldeia, para as diferentes idades e sexos. Esta análise foi realizada utilizando as duas escalas já referidas. Os resultados foram apresentados em termos de percentagens.

Foi também realizada uma análise estatística utilizando o $\mathrm{X}^{2}$ para conseguir testar as seguintes oito hipóteses:

- 1aH0: O nível de auto-estima das raparigas é independente do local onde moram.
- 2ª H0: O nível de auto-estimados rapazes é independente do local onde moram.

- 3aH0: O nível de auto-estima das raparigas de 14 anos é independente do local onde moram.

- 4르0: O nível de auto-estima dos rapazes de 14 anos é independente do local onde moram.

- 5oH0: O nível de auto-estima das raparigas de 15 anos é independente do local onde moram.

- 6aH0: O nível de auto-estima dos rapazes de 15 anos é independente do local onde moram.

- 7äH0: O nível de auto-estima das raparigas de 16 anos é independente do local onde moram.

- 8a H0: O nível de auto-estima dos rapazes de 16 anos é independente do local onde moram.

\section{Apresentação dos resultados}

Dos resultados obtidos, pela análise de freqüências, citamos os seguintes nas escala de zero a cinco (0-5) para a amostra total:

- do total de 486 de indivíduos do sexo masculino, nem um único teve frequências (0\%) na escala 5 (o nível mais baixo de auto-estima) enquanto que $1,65 \%$ dos indivíduos do sexo feminino se classificaram a este nível;

- as frequências mais elevadas da escala 0 pertenceram ao sexo masculino com a idade de 16 anos a habitar na cidade $(28,89 \%)$ e as mais baixas também ao sexo masculino com a idade de 14 anos e a habitar na aldeia (13,33\%);

- as freqüências mais elevadas da escala 1 pertenceram ao sexo feminino com a idade de 16 anos e a habitar na cidade $(38,89 \%)$ e as mais baixas também ao 
sexo feminino com 14 anos e a habitar na aldeia $(15,49 \%)$;

- as freqüências mais elevadas da escala 2 pertenceram ao sexo masculino com 15 anos e a habitar na aldeia $(36,96 \%)$ e as mais baixas ao sexo feminino com 16 anos e a habitar na cidade (19,44\%);

- as freqüências mais elevadas da escala 3 pertenceram ao sexo feminino com 16 anos e a habitar na cidade $(26,67 \%)$ e as mais baixas também ao sexo feminino com 15 anos e a habitar na aldeia ( $10,53 \%)$;
- asfreqüências mais elevadas da escala 4 pertenceram ao sexo masculino com 16 anos e a habitar na cidade ( $15,56 \%)$ e as mais baixas também ao sexo masculino com 14 anos e a habitar na cidade $(1,23 \%)$;

- as freqüências mais elevadas na escala 5 pertenceram ao sexo feminino com 14 anos e a habitar na aldeia $(4,23 \%)$ e as mais baixas ao sexo masculino em qualquer idade e qualquer que seja a sua residência ( $0 \%)$.

\section{CIDADE}

\begin{tabular}{|l|c|c|c|}
\hline & ALTA & MÉDIA & BAIXA \\
\hline 14 ANOS & & & \\
RAPAZES & $55,56 \%$ & $43,21 \%$ & $1,23 \%$ \\
\hline RAPARIGAS & $49,44 \%$ & $47,19 \%$ & $3,37 \%$ \\
\hline 15 ANOS & & & \\
\hline RAPAZES & $52,17 \%$ & $44,93 \%$ & $2,90 \%$ \\
\hline RAPARIGAS & $52,00 \%$ & $40,00 \%$ & $8,00 \%$ \\
\hline 16 ANOS & & & \\
\hline RAPAZES & $51,11 \%$ & $33,33 \%$ & $15,56 \%$ \\
\hline RAPARIGAS & $63,89 \%$ & $33,33 \%$ & $2,78 \%$ \\
\hline
\end{tabular}

Dos resultados obtidos citamos os seguintes na escala de auto-estima - Alta, Média e Baixa:

- as freqüências mais elevadas da escala Alta pertenceram ao sexo feminino com a idade de 16 anos e a habitar na cidade $(63,89 \%)$ versus sexo feminino com a idade de 16 anos e a habitar na aldeia (44\%) e as mais baixas também ao sexo feminino com a idade de 14 anos e a habitar na aldeia $(30,99 \%)$ versus sexo feminino com a idade de 14 anos e a habitar na cidade $(49,44 \%)$;

- as freqüências mais elevadas da escala Média pertenceram ao sexo feminino com a idade de 14 anos e a habitar na aldeia (56,34\%) versus sexo feminino com a idade de 14 anos e a habitar na cidade

\begin{tabular}{|c|c|c|}
\multicolumn{3}{|c}{ ALDEIA } \\
\hline ALTA & MÉDIA & BAIXA \\
\hline & & \\
\hline $43,33 \%$ & $51,67 \%$ & $5,00 \%$ \\
\hline $30,99 \%$ & $51,67 \%$ & $12,68 \%$ \\
\hline & & \\
\hline $42,39 \%$ & $55,43 \%$ & $2,17 \%$ \\
\hline $56,84 \%$ & $36,84 \%$ & $6,32 \%$ \\
\hline $49,25 \%$ & $47,76 \%$ & $2,99 \%$ \\
\hline $44,00 \%$ & $54,67 \%$ & $1,33 \%$ \\
\hline
\end{tabular}

$(47,19 \%)$ e as mais baixas simultaneamente ao sexo feminino masculino com 16 anos a habitar na cidade $(33,33 \%)$ versus sexo feminino com 16 anos a habitar na aldeia (54,67\%) e o sexo masculino com 16 anos a habitar na aldeia $(47,76 \%)$;

- as freqüências mais elevadas da escala Baixa pertenceram ao sexo masculino com 16 anos a habitar na cidade(15,56\%) versus sexo masculino com 16 anos a habitar na aldeia $(2,99 \%)$ e as mais baixas também ao sexo masculino com 14 anos a habitar na cidade $(1,23 \%)$ versus sexo masculino com 14 anos a habitar na aldeia (5\%).

Da análise estatística utilizando $X^{2}$ obtivemos os seguintes resultados: 
- 1a H0: O nível de auto-estima das raparigas é independente do local onde moram.

\begin{tabular}{llll}
\hline TEST ST ATISTIC & VALUE & DF & PROB \\
\hline PEARSON CHI-SQUARE & 4,730 & 2 & 0,094 \\
\hline
\end{tabular}

O valor obtido, 4,730, é inferior ao valor tabelado $(5,99)$ para dois graus de liberdade e um intervalo de confiança de $95 \%$, pelo que se aceita a hipótese.

- 2-H0: O nível de auto-estima dos rapazes é independente do local onde moram.

\begin{tabular}{llll}
\hline TEST ST ATISTIC & VALUE & DF & PROB \\
\hline PEARSON CHI-SQUARE & 5,755 & 2 & 0,056 \\
\hline
\end{tabular}

Ainda que o valor obtido, 5,755 , se aproxime muito do valor tabelado $(5,99)$ ainda é inferior, para dois graus de liberdade e um intervalo de confiança de $95 \%$, pelo que se aceita a hipótese.

- 3ä0: O nível de auto-estima das raparigas de 14 anos é independente do local onde moram.

\begin{tabular}{llll}
\hline TEST ST ATISTIC & VALUE & DF & PROB \\
\hline PEARSON CHI-SQUARE & 10,540 & 2 & 0,005 \\
\hline
\end{tabular}

O valor obtido, 10,540, é superior ao valor tabelado $(9,21)$ para dois graus de liberdade e um intervalo de confiança de $99 \%$, pelo que se rejeita a hipótese. Existe assim uma dependência muito significativa $(\mathrm{P}<0,01)$.

- 4aH0: O nível de auto-estima dos rapazes de 14 anos é independente do local onde moram.

\begin{tabular}{llll}
\hline TEST STATISTIC & VALUE & DF & PROB \\
\hline PEARSON CHI-SQUARE & 4,537 & 2 & 0,103 \\
\hline
\end{tabular}

O valor obtido, 4,537, é inferior ao valor tabelado $(5,99)$ para dois graus de liberdade $e$ um intervalo de confiança $95 \%$, pelo que se aceita a hipótese.

- 5aH0: O nível de auto-estima das raparigas de 15 anos é independente do local onde moram.

\begin{tabular}{llll}
\hline TEST ST ATISTIC & VALUE & DF & PROB \\
\hline PEARSON CHI-SQUARE & 0,623 & 2 & 0,733 \\
\hline
\end{tabular}

O valor obtido, 0,623, é inferior ao valor tabelado $(5,99)$ para dois graus de liberdade e um intervalo de confiança de $95 \%$, pelo que se aceita a hipótese.

- 6ㄹ0: O nível de auto-estima dos rapazes de 15 anos é independente do local onde moram.

\begin{tabular}{llll}
\hline TEST ST ATISTIC & VALUE & DF & PROB \\
\hline PEARSON CHI-SQUARE & 2,252 & 2 & 0,324 \\
\hline
\end{tabular}

O valor obtido, 2,252, é inferior ao valor tabelado $(5,99)$ para dois graus de liberdade e um intervalo de confiança de $95 \%$, pelo que se aceita a hipótese.

- 7äH0: O nível de auto-estima das raparigas de 16 anos é independente do local onde moram.

\begin{tabular}{llll}
\hline TEST ST ATISTIC & VALUE & DF & PROB \\
\hline PEARSON CHI-SQUARE & 7,243 & 2 & 0,027 \\
\hline
\end{tabular}

O valor obtido, 7,243 , é superior ao valor tabelado $(5,99)$ para dois graus de liberdade e um intervalo de confiança de $95 \%$, pelo que se rejeita a hipótese.

Existe assim uma dependência significativa $(P<0,05)$. 
- 8H0: O nível de auto-estima dos rapazes de 16 anos é independente do local onde moram.

\begin{tabular}{llll}
\hline TESTSTATISTIC & VALUE & DF & PROB \\
\hline PEARSON CHI-SQUARE & 7,735 & 2 & 0,021 \\
\hline
\end{tabular}

O valor obtido, 7,735 , é superior ao valor tabelado $(5,99)$ para dois graus de liberdade e um intervalo de confiança de $95 \%$, pelo que se rejeita a hipótese.

Existe assim uma dependência significativa $(P<0,05)$.

Os resultados obtidos pela análise estatística de $X^{2}$, vieram confirmar quase totalmente (com excepção do sexo masculino com 14 anos a habitar na cidade versus sexo masculino com 14 anos a habitar na aldeia) os resultados obtidos pela análise da escala - Alta; Média e Baixa - assim como os resultados obtidos pela escala de 0 a 5 .

\section{Discussão e conclusões}

O facto de certos adolescentes residirem na cidade ou na aldeia tem repercussões no seu nível de auto-estima. Na amostra estudada essas diferenças notam-se nos escalões etários dos 16 anos para ambos os sexos e, de uma forma mais evidente, nas raparigas de 14 anos que moram na aldeia e possuem freqüências inferiores de auto-estima-Alta e superiores da auto-estima-Baixa em relação às da cidade no mesmo escalão etário. Se analisarmos os dados em relação à escala de 0 a 5 , verificámos que na cidade, para a escala 0 e 1 (que são representativos de auto-estima Alta) as raparigas (14 anos) apresentam freqüências de 19,10\% e $30,34 \%$ respectivamente contra $15,49 \%$ e $15,49 \%$ nas da aldeia.

Em relação à escala de 4 a 5 (que são representativos de auto-estima Baixa) as da cidade (14 anos) apresentam freqüências, de $3,37 \%$ e $0 \%$ respectivamente contra $8,45 \%$ e $4,23 \%$ nas da aldeia. Em conclusão, as raparigas de 14 anos da cidade, nesta amostra, possuem freqüências mais elevadas de autoestima global do que as raparigas da aldeia.

As raparigas de 16 anos da cidade possuem, também, freqüências mais elevadas de auto-estima Alta do que as da aldeia, mas curiosamente, também apresentam freqüências mais elevadas de auto-estima Baixa, 2,78\% na cidade contra $1,33 \%$ na aldeia. É de notar que este valor de 1,33\% para auto-estima Baixa é obtido à custa dos resultados das aldeias de Vila Real, pois Chaves e Mirandela apresentaram freqüências de $0 \%$ de auto-estima Baixa. $\mathrm{Na}$ aldeia, curiosamente, as percentagens de autoestima Alta, no caso de Mirandela, coincidem com as de Média auto-estima (50\% para ambas) e no caso de Chaves quase coincidem (Alta-46,15\% e Baixa-53,85\%).Isto transmite-nos a ideia de que a amostra da aldeia, neste escalão, comporta-se de uma forma homogénea, como se possuísse uma identidade comum, ao contrário da cidade em que se verificam extremos (no caso de Chaves a Média auto-estima chega a coincidir com a auto-estima Baixa $-22,22 \%$ para ambas). Se tivermos em conta que a auto-estima esta sempre dependente de uma variedade de factores do meio ambiente (biológicos, familiares, socioculturais, etc.) em variados momentos (Juhasz, 1985), talvez possamos atribuir esses resultados ao facto de na aldeia certos factores serem mais comuns e incidentes e em essência diferentes da cidade. Mas mesmo assim, surge-nos outra questão - porque é que isto se verifica somente neste escalão etário? Pararesponder a estapergunta teria de se proceder a um estudo mais aprofundado e com mais variáveis envolvidas.

Os rapazes de 16 anos da cidade também possuem, à semelhança das raparigas, frequências mais elevadas de auto-estima Alta do que os da aldeia assim como freqüências mais elevadas de auto-estima Baixa. De alguma forma parece que na cidade, para os jovens de 16 
anos, freqüências mais altas de auto-estima global encontram-se associados à freqüências mais baixas de auto-estima global. Isto quer dizer que, embora na aldeia (para estas idades) existam menos indivíduos com auto-estima alta, também é verdade que existem menos indivíduos com auto-estima baixa, ao contrário da cidade (essas diferenças reflectem-se na auto-estima Média, que é maior na aldeia do que na cidade, para ambos os sexos). De alguma forma, semeIhante ao que acontecia com as raparigas de 16 anos, para as freqüências de auto-estima Baixa dos rapazes na aldeias, só contribuem os indivíduos das aldeias de Mirandela (8,70\%), contribuindo Chaves e Vila Real com $0 \%$.

Na nossa amostra as raparigas de 14 anos revelam uma auto-estima global inferior aos rapazes da mesma idade, o que se inverte aos quinze e dezasseis anos de idade. Neste grupo etário são os rapazes que apresentam uma auto-estima global mais baixa. Isto vai de alguma forma ao encontro das afirmações de Marshall e Harper (1991) que argumentam que a auto-estima das raparigas quando com parada com a dos rapazes diminui por altura da puberdade. Estes resultados podem ser explicados em parte pelos factores relacionados com o processo da maturação biológica, uma vez que estas têm um avanço relativamente aos rapazes. As implicações desta diferença necessitam ser exploradas tomando em consideração os processos de organização e integração social dos membros de ambos os sexos, nomeadamente no que diz respeito aos papéis sociais que Ihes vão sendo atribuídos ao longo dos anos. Por outras palavras, é preciso estudar as diferenças entre as obrigações socioculturais a serem assumidas nesta faixa etária por ambos os sexos e que vão para além dos conflitos inerentes às mudanças físicas e à sexualidade emergente. Segundo Savin-Williams e Jaquish (1981), talvez as raparigas sejam mais sensíveis e críticas em relação às suas percepções do que os rapazes. Por outro lado, se a adolescência é de facto uma fase em que devido a factores hormonais os sexos se diferenciam, também poderá ser um período em que a diferen- ça de auto-estima entre os sexos pode estar relacionada com os factores de socialização.

O nosso estudo valida os que argumentam que existem algumas diferenças nas freqüências de auto-estima dos adolescentes consoante a sua residência. Devido ao facto de isso poder ser resultante de uma miríade de factores, será necessário uma série de estudos para conseguir isolar e controlar esses factores no desenho da investigação. No caso particular deste estudo, seria importante repeti-lo utilizando desta vez escolas de grandes metrópoles como, por exemplo Lisboa ou Porto, de forma a podermos comparar os níveis de auto-estima dos jovens e assim verificar se a dimensão do local residencial tem ou não influência nas diferenças de auto-estima dos jovens das aldeias ou das pequenas cidades. Como a valores baixos de auto-estima global se encontram geralmente associados a sentimentos de depressão, infelicidade, tristeza e inferioridade (Rosenberg, 1989), seria também importante estudar em que medida estes sentimentos assolam os nossos jovens. Ainda gostaríamos de ir mais longe, pesquisando a correlação da auto-estima com fenómenos sociais como o suicídio, delinquência, toxicodepen-dência, etc... O campo de pesquisa revela-se extremamente vasto e para quem trabalha com jovens, a pesquisa na área da auto-estima revela-se aliciante e fundamental para o apoio à resolução dos seus problemas.

\section{BIBLIOGRAFIA}

ABERNATHY, T. J., MASSAD, L., \& ROMANO-DWYER, L. (1995). The Relationship Between Smoking and Self-Esteem. Adolescence, 30, 900-907.

BALDWIN, M.K., \& COURNEYA, K.S. (1997). Exercise and self-esteem in breast cancer survivors: An application of the exercise and self-esteem model. Journal of Sport \& Exercise Psychology, 19,347-359. 
BATISTA, P. (1995). Satisfação com Imagem Corporal e Auto-estima: Estudo Comparativo de Adolescentes envolvidas em diferentes níveis de actividade física. Tese de Mestrado. Faculdade Ciencias do Desporto e Educação Física, Universidade do Porto.

BEER, J. (1987). Depression and self-esteem of teachers. Psychological Reports, 60, 1097-1098.

BHAATI, B., DEREZOTES, D.,KIM, S.,SPETCH, $H$. (1989). The association between child maltreatement and self-esteem. In A.M. Mecca, N.J. Smelser, and J.Vasconcellos, (Ed.). The social importance of selfesteem, pp. 24-62. Los Angeles: The Regents of the University of California.

BURNS, R., (1979). The self-Concept: Theory, measurement, development and behaviour. London: Longman.

CALFAS, K., TAYLOR, W. (1994). Effects of physical activity in psychological variables in adolescents. Pediatric Exercise Science, 6,406-423.

CATE, R., SUGAWARA, A. (1986). Sex role orientation and dimensions of self-esteem among adolescents. Sex Roles, 15, 145-158.

CHIU, L. (1990). The relationship of career goal and self-esteem among adolescents. Adolescence, XXV, 593-597.

COELHO, E. e RAPOSO, J. (no prelo). A autoestima em atletas de alta competição de nacionalidade portuguesa. UT AD-SDE.

COOPERSMITH, S. (1959). A method for determining types of self-esteem.Journal of Abnormal and Social Psychology, 59,87-94.

CROCKENBERG, S. B. SOBY, B. A. (1989). Self-Esteem and Teenage Preqnancy . In A.M. Mecca, N.J. Smelser, and J.Vasconcellos, (Ed.). The social importance of self-esteem, pp. 125-161. Los Angeles: The Regentsof the University of California.
DALY, J.A., KREISER, P.O., \&ROGHAAR, L.A. (1994). Ouestion-asking comfort: Explorations of the demography of communication in the eight grade classroom. Communication Education, 43, 27-42.

FOX, K., CORBIN, C. (1989). The physical perception profile: Development and validation. Journal of Sport and Exercise Psychology, 11,408-430.

FOX,K., R. (1988). The Self-Esteem Complex and Youth Fitness . Quest. 40, 230-246.

GODIN,P., LEVAL,N. (1993). Self-esteem and achievement in sport. Actas do 8 congresso mundial de psicologia desportiva, psicologia do desporto: Uma perspectiva integrada, pp. 558-561.

GREEN, A., REED, E. (1992). Social context differences in the relation between self-esteem and self-concept during late adolescence. Journal of Adolescence Research, 7, 266-282.

GREENE, A. L., REED, E. (1992). Social context differences in the relation between self-esteem and self-concept during late adolescence. Journal of Adolescence Research, 7, 266-282.

HARPER, J., MARSHALL, E. (1991). Adolescents and their relationship to selfesteem. Adolescence, 26, 799-808.

HARTER, S. (1983). Development perspectives on the self-esteem. In E. M. Hetherington (Ed.) Handbook of Child Psychology, pp.275-385. N. Y.: Wiley.

HAYWOOD, K. (1986). Sports competition and its influence on self-esteem development. Adolescence, 34, 861-869.

HINES, S., GROVES, D. (1989). Sports competition and its influence on self-esteem developme nt. Adolescence, 34, 861-869.

JAQUISH, G., SAVIN-WILLIAMS, R. (1981). Biological and ecological factors in the expression of adolescent self-esteem. Journal of Youth and Adolescence, 10, 473-485. 
JUHASZ, A. (1985). Measurement self-esteem in early adolescents. Adolescence, XX, 877-893.

JUHASZ, A. (1989). Significant others and self-esteem: Methods for determining who and why. Adolescence, XXVI, 581-593.

KALTIKANGAN-JAVINEN, L. (1992). Self-esteem as a predictor of future school achievement. European Journal of Psychology of Education, 7,123-130.

KINCEY, J., AMIR, z., GILLEPSIE, B., CARLETON, E., andTHEAKER, T. (1993). A study of self-esteem, motivation and perceived barriers to participation in sport and exercise among secondary school pupils. Health Education Journal, 52, 241-245.

LACKOVIC-GRGIN, K., DEKOVIC, M. (1990). The contribution of significant others to adolescents' self-esteem. Adolescence, XXV, 839-846.

LOPES, D. (1996). Aptidão física e auto-estima: Um estudo em adultos idosos dos dois sexos do concelho de Matosinhos envolvidos num programa de actividades físicas regulares. Dissertação de Mestrado, Universidade do Porto, FCDEF.

PADIN, M. A., LERNER, R. M., SPIRO III, A. (1981). Stability of body attitudes and self-esteem in late adolescents. Adocescence, XVI, 371-384.

PECK, D. (1981). Adolescent self-esteem, Emotional Learning Disabilities, and Significant Others. Adolescence, XVI, 443445.

PEIXOTO, L.,MESQUITA, A. (1990). Aauto-estima, o nível intelectual e o sucesso escolar. Revista Portuguesa de Educação, 3, 8799.

PONTE, A., PORTUGAL, S., BARROSO, R., NEVES, P. (1991). Influência do contexto sóciodesportivo na formação do auto-concei- to. In FCDEF-UP (Eds.). As Ciências e a Prática Desportiva, Desporto na Escola, Desporto de Reeducação e Reabilitação, pp. 207-211, Porto:
Universidade do Porto.

PRAWAT, R.S., GRISSOM, S. \& PARISH, T. (1979). Aftective development in children, grades 3 through 12. The Journal of Genetic Psychology. 135, 37-49.

RAPOSO, J., CARVALHO, M. (1995). Normas gerais para a elaboração de trabalhos escritos. Vila Real. UTAD-SDE.

RICHMAN, C. L., CLARK, M. L., \& BROWN, K. P. (1985). General and specific self-esteem in late adolescents students: Race x gender x SES effects. Adolescence, XX, 555-566.

ROSENBERG, M. (1989). Society and the adolescent self-image. Connecticut: Wesleyan University Press.

SAVIN-WILLLAMS, R., JAQUISH, G. (1981). Biological and ecological factores in the expression if adolescent self-esteem. Journal of Youth and Adolescence, 10,77-81.

SCHILLING, D. (1986). Self-esteem: Concerns, strategies, resources. Academic Therapy, 21,301-307.

SCHNEIDERMAN, L., FURMAN, W., J. (1989). Self-esteem and chronicwelfare dependency. In A.M. Mecca, N.J. Smelser, and J.Vasconcellos, (Ed.). The social importance of self-esteem, pp.24-71. Los Angeles: University of California Press.

SERRA, A. (1986). A importância do autoconceito. Psiquiatria Clínica, 7, 57-66.

SHAVELSON, R., HUBNER, J., \& STANTON, G. (1976). Self-concept: Validation of construct interpretations. Review of Educational Research, 46, 407-441.

SIMON, W.E. (1972). Some sociometric evidence for validity of Coopersrnith's self-esteem inventory. Perceptual and Motor Skills, 34, 93-94.

SKAGER, R., KERST, E. (1989). Alcohol and drug use and self-esteem: A psychological perspective. In A.M. Mecca, N.J. Smelser, and J.Vasconcellos, (Ed.). The social importance of self-esteem, pp.248-286. Los Angeles: The Regents of the University of California. 
SMELSER, F. (1989). Self-esteem and social problems: An introduction . In A.M. Mecca, N.J. Smelser, and J.Vasconcellos, (Ed.). The social importance of self-esteem, pp. 1-23. Los Angeles: The Regents of the Un iversity of California.

SMITH, R.B., TEDESCHI, J.T., BROWN, R.C., and LINDSKOLD, S. (1973). Correlations between trust, self-esteem, sociometric choice and internal-external control. Psychological Reports, 32, 739-743.

SULLIVAN, P.F., KESSLER, A.C.,\&KENDLER, K.S. (1998). Latent class analysis of lifetime depressive symptoms in the National Comorbidity Survey. American Journal of Psychiatry, 155, 1398-1407.

VASCONCELLOS, J. (1989). Preface. In A.M. Mecca, N.J. Smelser, and J.Vasconcellos, (Ed.). The social importance of self-esteem, pp. xi-xxi. Los Angeles: The Regents of the University of California.

WALSH, A.,SUGAWARA, A.(1991). Self-esteem and sexual behavior: Exploring gender differences. Sex Roles, 25, 441-451.

WATKINS, D.,DMAWAN, M. (1989). Dowe need to distinguish the constructs of self-concept and self-esteem?.Journal of Social Behavior and Personality, 4, 555-562.
WEISS, M. (1987). Self-Esteem and achievement in children's sport and physical activity. In D, Gould, M. Weiss (Eds.). Advances in pediatric sport sciences, pp.87-119 (Vol.11), Champaign: Human Kinetics.

WEISS, M. (1993). Psychological effects of intensive sport participation on children and youth: Self-esteem and motivation. In B. Cahill, A. Pearl (Eds.) Intensive Participation in Children's Sports, pp.39-69, Human Kinetics.

WHITEHEAD, J. (1995). A study of children's physical Self-Perceptions using an adapted physical Self-Perception Profile Questionnaire. Pediatric Exercise Science, 7,132-151.

WYLIE, A. (1979). The Self Concept . Vo1.2. Lincoln, Nebraska: University of Nebraska Press.

YOUNGS, George A., RATHGE, RICHARD, MULLIS, RON, MULLIS, ANN (1990). Adolescent stress and self-esteem. Adolescence, XXV, 333-341.

ZIEMAN, G.,BENSON, G. (1983). Delinquency: the Role of self-esteem and social values. Journal of Youth and Adolescence, 12, 489-500. 